\title{
Linking high harmonics from gases and solids
}

\author{
G. Vampa ${ }^{1}$, T. J. Hammond ${ }^{1}$, N. Thiré2 ${ }^{2}$ B. E. Schmidt ${ }^{2}$, F. Légaré2 ${ }^{2}$ C. R. McDonald ${ }^{1}$, T. Brabec ${ }^{1} \&$ P. B. Corkum ${ }^{1,3}$
}

When intense light interacts with an atomic gas, recollision between an ionizing electron and its parent ion ${ }^{1}$ creates high-order harmonics of the fundamental laser frequency ${ }^{2}$. This sub-cycle effect generates coherent soft $\mathrm{X}$-rays ${ }^{3}$ and attosecond pulses ${ }^{4}$, and provides a means to image molecular orbitals ${ }^{5}$. Recently, high harmonics have been generated from bulk crystals ${ }^{6,7}$, but what mechanism $^{8-12}$ dominates the emission remains uncertain. To resolve this issue, we adapt measurement methods from gas-phase research ${ }^{13,14}$ to solid zinc oxide driven by mid-infrared laser fields of 0.25 volts per ångtröm. We find that when we alter the generation process with a second-harmonic beam, the modified harmonic spectrum bears the signature of a generalized recollision between an electron and its associated hole ${ }^{11}$. In addition, we find that solidstate high harmonics are perturbed by fields so weak that they are present in conventional electronic circuits, thus opening a route to integrate electronics with attosecond and high-harmonic technology. Future experiments will permit the band structure of a solid ${ }^{15}$ to be tomographically reconstructed.

Fifty years ago, Keldysh introduced an important idea. Treating both gases and solids on the same footing, he showed that strong-field ionization-valence-band to conduction-band transitions in solidscan be approximated by tunnelling ${ }^{16}$. Over the following decades, research on the interaction of intense pulses with solids and gases has diverged. Much of the solid-state research has been motivated by laser materials processing ${ }^{17}$. A great deal of the gas-phase research has been motivated by high-harmonic generation ${ }^{18,19}$, a process initiated by tunnelling and completed by the recollision of a highly energetic electron with its associated ion (hole). However, the deep similarity in their response to strong fields has been largely ignored. Now that high harmonics have been measured from $\mathrm{ZnO}$ (ref. 6) and other condensed media ${ }^{7,20}$, it is important to reconsider the relation between the gas-phase and solid-state mechanisms.

Individual ionizing atoms provide two sources of harmonics. Expressed in the language of solid-state studies, there is a 'single-band' (intraband) contribution, in which the recently tunnelled electron wave packet undergoes oscillatory motion in the laser field. Here the nonlinearity enters through the step-wise nature of tunnel ionization ${ }^{21}$. In addition, there is a 'two-band' (interband) source. In atoms, this source is interpreted in terms of the ionized electron recolliding and recombining with its associated hole (parent ion), emitting a highenergy photon in the process. In gases, the former source dominates the low harmonics while the latter source dominates high-harmonic generation. Electrons and holes in solids exhibit similar mechanisms as they move in, and between, the conduction and valence bands ${ }^{10,11}$. However, in a solid, the electron's interaction with the lattice makes its oscillation nonlinear; this is a new source of intraband emission ${ }^{8}$, and leads to a generalized recollision ${ }^{11}$. The relative contribution of these intra- and interband mechanisms and their interplay is important theoretically ${ }^{8-12}$ and experimentally ${ }^{7}$. The unresolved issue is under what circumstances does one physical mechanism dominate production of high harmonics in solids.

To resolve this issue, we adapt a method of gas-phase research to solids ${ }^{13}$. Using a mid-infrared fundamental laser to generate odd harmonics in $\mathrm{ZnO}$, we perturb the process with a very weak secondharmonic beam, thereby producing even harmonics. The strength of the even harmonics modulates as the second harmonic is delayed relative to the fundamental. The phase of the modulation for each harmonic order determines the spectral phase of the emitted harmonic beam and characterizes the generation mechanism. Using photons with energies well below that of resonance $\left(h v \approx 0.1 E_{\mathrm{g}}\right.$, where $E_{\mathrm{g}}$ is the bandgap of the solid), we find that a generalized recollision between the electron and its associated hole plays the dominant role in high-harmonic emission from $\mathrm{ZnO}$ at the field strength of our experiment, as it does in gases. By 'generalized recollision' we mean that between tunnelling and recollision the electron and hole move on their respective bands, as discussed in the Methods section. Furthermore, as we increase the relative second-harmonic intensity, for each intensity range we find solid-state behaviour that closely mirrors the atomic response. There is, however, one possible exception. As described in the Methods section, in gases there are so-called long and short trajectory contributions, which lead to differently diverging radiation. Although we have searched for them, we have not resolved long-trajectory harmonics in our experiment. However, even this may be the same in gases since long-trajectory harmonics are hard to observe with infrared drivers.

To understand the experiment it is useful to think of high-harmonic generation as a balanced interferometer. As illustrated in Fig. 1a for atomic recollision, the electron (and hole) wave packets created on subsequent half-cycles of the driving field propagate on equal but oppositely directed trajectories (equal-length arms of an interferometer), creating sub-cycle bursts of short-wavelength radiation when they recollide. With each subsequent pulse having opposite phase because of the oppositely directed collision, when the pulses are spectrally resolved, only odd harmonics constructively interfere. Adding a weak second-harmonic field unbalances the trajectories (interferometer), adding a small phase to one arm while removing it from the other, thereby producing even harmonics. For $\mathrm{ZnO}$, because of the low reduced carrier mass near the band centre, low-photon-energy harmonics are very sensitive to weak second-harmonic fields. In a recollision process, the phase that maximally unbalances the interferometer differs between harmonic orders. While illustrated for recollision, the interferometer analogy also applies to intraband oscillations: here, the asymmetry originates from the slightly different current generated in two successive half-cycles, as a result of the electron reaching higher (lower) momentum when it propagates in the left (right) side of the Brillouin zone. Because harmonics are emitted as the electron moves, they are all phased together ${ }^{10}$. The second harmonic perturbs all of them equally and simultaneously, so the phase that maximally unbalances the interferometer is the same for all harmonics. This different behaviour allows us to discriminate between recollision and non-recollision based mechanisms.

Experimentally, high harmonics are produced by focusing the fundamental and the second harmonic of mid-infrared laser pulses (3.76 $\mu \mathrm{m}$ central wavelength) in a, 500-nm $\mathrm{ZnO}$ single crystal at an intensity of $0.85 \mathrm{TW} \mathrm{cm}^{-2}\left(0.25 \mathrm{~V}^{-1}\right)$ in the crystal. The two colours

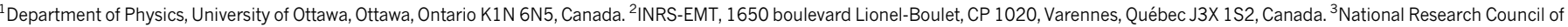
Canada, Ottawa, Ontario K1A OR6, Canada. 
a
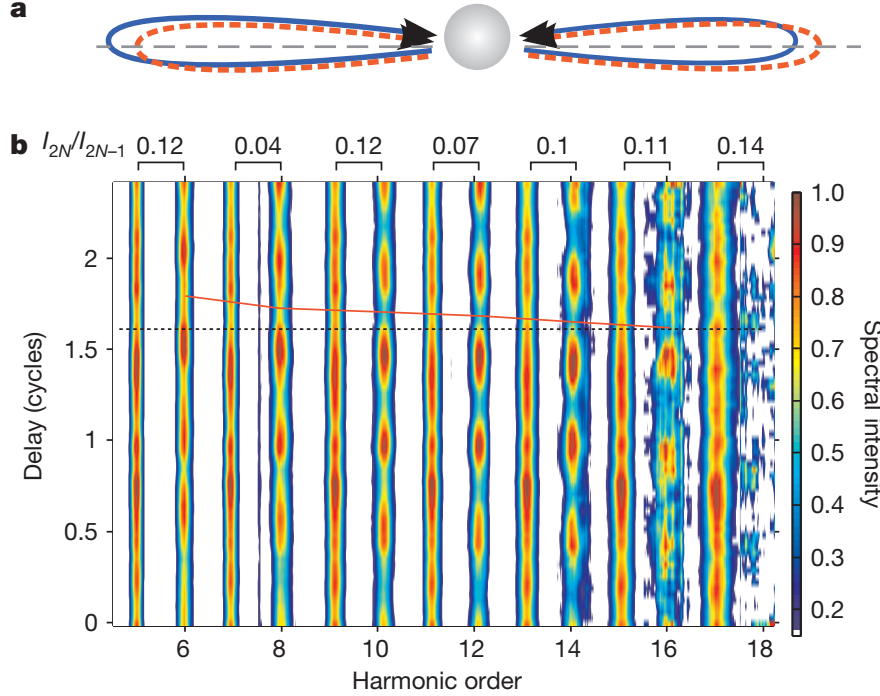

Figure $1 \mid$ Realization of the in situ experiment. a, Pictorial representation of the electron's trajectories. In the fundamental field alone, the electron travels symmetrically to the left and to the right of the ion (central grey sphere) during two successive half-cycles (red dashed lines). The addition of the second harmonic lengthens one trajectory while shortening the other (solid blue lines), thereby breaking the symmetry and producing even harmonics. b. Measured high-harmonic spectra versus the delay between the two colours; the spectral intensity is colour-coded (key at right). The even harmonic intensity modulates every half-cycle. The odd harmonics also modulate, but the modulation is much weaker. The phase of the even harmonic modulation depends on the harmonic order, as evidenced by the red solid line relative to the black reference line. (The red line links the minima in the modulation of the even harmonic intensity.) Each harmonic order is normalized separately. The relative intensity between even-order and adjacent odd-order harmonics is given at the top $\left(I_{2 N} / I_{2 N-1}\right)$. The delay of the second harmonic is defined in cycles of the fundamental.

(that is, fundamental and second harmonic) have parallel polarizations (details of the experimental set-up are given in the Methods section).

Figure $1 \mathrm{~b}$ shows the harmonic spectrum versus the relative phase between the two colours, for a second-harmonic intensity $9 \times 10^{-6}$ times that of the fundamental. Each harmonic order is independently normalized; the relative intensity between adjacent orders $\left(I_{2 N} / \mathrm{I}_{2 N-1}\right)$ is reported at the top of the graph ( $N$ is an integer that identifies the harmonic order). The weak even-harmonic signal modulates with an order-dependent phase $\Phi_{\text {osc }}(2 N)$ as a function of delay. Similar behaviour is measured for atomic harmonics ${ }^{13}$, where $\Phi_{\text {osc }}$ is used to extract the spectral phase of each even harmonic or, equivalently, the emission time of the attosecond pulses. Here, we use $\Phi_{\text {osc }}$ to establish the origin of solid-state harmonics.

Figure 2 shows $\Phi_{\text {osc }}$ plotted as a function of harmonic order (black circles) up to the cut-off of our spectrograph. We use an unmeasured constant phase to position the experimental data (this constant phase is measurable using nonlinear optical methods). The theoretical values of $\Phi_{\text {osc }}$ calculated from a two-band model are also plotted for the intraband (purple line) and interband (blue line) sources, and for their combined emission (gold dashed line); the calculations are described in the Methods section. We find that the experiment is consistent with the theoretical prediction (Extended Data Fig. 6) that interband harmonics dominate in $\mathrm{ZnO}$ for the field strength used (we note that recent work has found evidence for the dominance of intraband oscillations for $\mathrm{THz}$ excitation ${ }^{7}$ ). In the Methods section we investigate theoretically how our results scale with the field strength of the fundamental.

The slope of the curves in Fig. 2 is determined by both the intensity of the fundamental and the band structure of the crystal. Crystal dispersion has two major consequences: first, the electron-hole pair accumulates more phase than in a gas ( $\sim 10$ times for the short trajectories), making it easier to break the symmetry with the second-harmonic

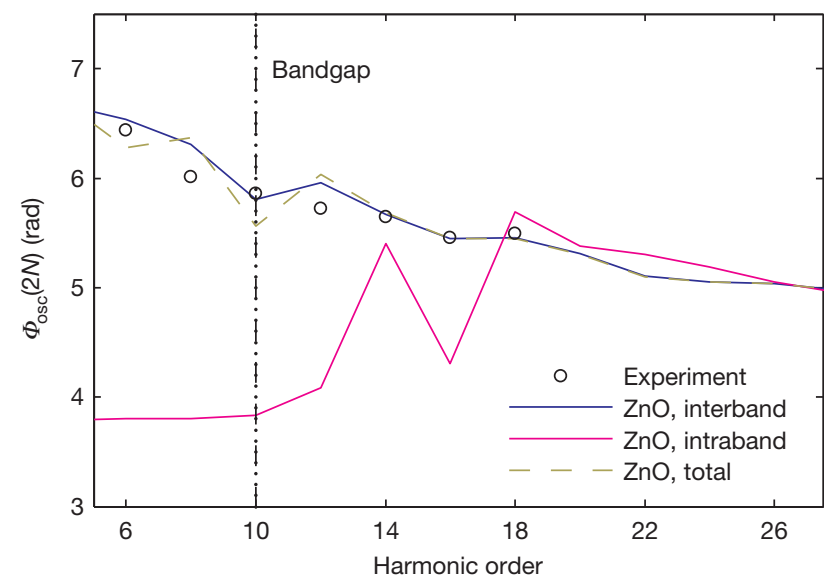

Figure $2 \mid$ Comparison with theory. The phase of the modulation of the even harmonics is extracted from Fig. 1 (black circles) and compared to the simulated intraband (purple line) and interband (blue line) phase. The simulated phase for their combined emission (intraband plus interband, yellow dashed line) agrees with the interband emission, in agreement with interband harmonics being significantly stronger than intraband ones (Extended Data Fig. 6).

field. This is why we require second-harmonic intensities of only $\sim 10^{-5}$ times that of the fundamental. Second, crystal dispersion influences the duration of the high-harmonic pulses. We measure a harmonic chirp of $0.38 \mathrm{fs} \mathrm{eV}^{-1}$ at the 16th harmonic, corresponding to a train of pulses with duration of $1.7 \mathrm{fs}$ (before dispersion compensation) for a $\sim 2 \mathrm{eV}$ bandwidth. Wider bands and higher intensities will result in smaller chirps, thereby allowing attosecond pulse generation from solids.

As the second-harmonic intensity is increased, we move from perturbing to controlling harmonic emission. In gases, we first reach an intensity where $\Phi_{\text {osc }}$ is nearly out of phase for the even and odd harmonics ${ }^{13}$. Figure 3 a shows experimental results for $\mathrm{ZnO}$ using a second-harmonic intensity of $\sim 10^{-4}$ times that of the fundamental. The alternation between even and odd harmonics occurs when the second harmonic modulates the electron phase by $\Phi_{\mathrm{osc}}=\pi / 2$. For even higher second-harmonic field strengths (Fig. 3b), high-harmonic emission is substantially modified. In the highly asymmetric sum field, tunnelling only occurs once per laser cycle. This leads to simultaneous emission (or suppression) of even and odd harmonics-a behaviour well-studied for atomic gases ${ }^{14}$.

Taken together, our findings provide strong evidence that, for the field strength and laser wavelength of our experiment, a generalized recollision between an electron and its associated hole is the primary source of high harmonics in ZnO. Thus, 50 years after Keldysh's seminal paper ${ }^{16}$, we rebuild the connection linking the high-field response of gases and solids. But decoherence is a major difference between solids and gases. The occurrence of electron-hole recombination demonstrates the tolerance of the short-lived electron to dephasing. Although scattering times vary considerably between materials $\mathrm{s}^{22,23}$ and with excitation conditions ${ }^{24}$, our measurements suggest that highharmonic technology could have a broad impact in condensed media.

In this paper we have exploited our ability to manipulate the phase that the electron-hole pair acquires to identify the fundamental mechanism dominating harmonic generation in $\mathrm{ZnO}$. The phase also encodes information about the band structure of the material in which the electron travelled. By measuring $\Phi_{\text {osc }}$ as a function of crystal orientation, it will be possible to reconstruct a material's three-dimensional momentum-dependent band structure ${ }^{15}$. This all-optical technique will allow measurement of band structures where it is not possible to detect photoelectrons, for example in high-pressure experiments ${ }^{25}$.

Although we have not concentrated on it here, the tunnelling step determines the harmonic intensity. Our weak second-harmonic beam 


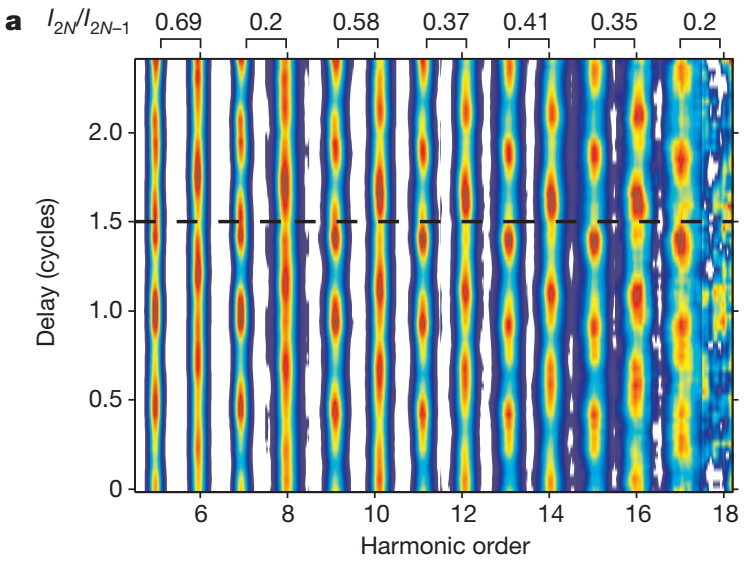

Figure $3 \mid$ From perturbing to controlling harmonic generation. a, When the intensity of the second harmonic reaches $1 \times 10^{-4}$ times that of the fundamental, the even and odd harmonics modulate out of phase. $\mathbf{b}$, Further increasing the intensity to $3 \times 10^{-3}$ times that of the fundamental, we find that

not only modifies the electron phase but also slightly alters tunnelling, thereby leading to oscillations of the odd harmonics with the delay between the two colours. The relative phase of the modulation between even and odd orders contains detailed information on the timedependent tunnelling rate ${ }^{26}$. Applying this analysis to solids will allow us to probe the sub-cycle dynamics of strong-field tunnelling in solids $s^{27,28}$-the essence of laser material modification ${ }^{17}$.

Finally, independent of the mechanism, we have demonstrated that a clear spectral signature can be imposed on a high-harmonic beam using a control beam having a peak electric field of $\sim 5 \mathrm{~V} \mathrm{~m}^{-1}$. Fields of this magnitude are present in electronic circuits ${ }^{29}$. Thus, we have bridged the gap between electronics and attosecond physics. If the sensitivity that we have demonstrated to perturbing fields can be transferred to silicon, it will be possible to record movies of working semiconductor electrical circuits or plasmons propagating in nano-plasmonic devices. Harmonics generated from a spatially complex circuit will diffract according to the instantaneous distribution of the internal fields. The control field can also be applied by electrodes on the crystal, thereby allowing manipulation of the harmonic beam with DC or pulsed electrical signals. Thus we have taken a significant step towards a new area of research, that of solid-state attosecond electronics ${ }^{30}$.

Online Content Methods, along with any additional Extended Data display items and Source Data, are available in the online version of the paper; references unique to these sections appear only in the online paper.

\section{Received 11 November 2014; accepted 27 April 2015.}

1. Corkum, P. B. A plasma perspective on strong field multiphoton ionization. Phys. Rev. Lett. 71, 1994-1997 (1993)

2. L'Huillier, A. \& Balcou, P. High-order harmonic generation in rare gases with a 1-ps 1053-nm laser. Phys. Rev. Lett. 70, 774-777 (1993).

3. Spielmann, Ch et al. Generation of coherent soft X-rays in the water window using 5-femtosecond laser pulses. Science 278, 661-664 (1997).

4. Goulielmakis, E. et al. Single-cycle nonlinear optics. Science 320, 1614-1617 (2008).

5. Itatani, J. et al. Tomographic imaging of molecular orbitals. Nature $\mathbf{4 3 2 , 8 6 7 - 8 7 1}$ (2004).

6. Ghimire, S. et al. Observation of high-order harmonic generation in a bulk crystal. Nature Phys. 7, 138-141 (2011)

7. Schubert, O. et al. Sub-cycle control of terahertz high-harmonic generation by dynamical Bloch oscillations. Nature Photon. 8, 119-123 (2014).

8. Kemper, F., Moritz, B., Freericks, J. K. \& Devereaux, T. P. Theoretical description of high-order harmonic generation in solids. New J. Phys. 15, 023003 (2013).

9. Higuchi, T., Stockman, M. I. \& Hommelhoff, P. Strong-field perspective on highharmonic radiation from bulk solids. Phys. Rev. Lett. 113, 213901 (2014).

10. Golde, D., Meier, T. \& Koch, S. High harmonics generated in semiconductor nanostructures by the coupled dynamics of optical inter- and intraband excitations. Phys. Rev. B 77, 075330 (2008).

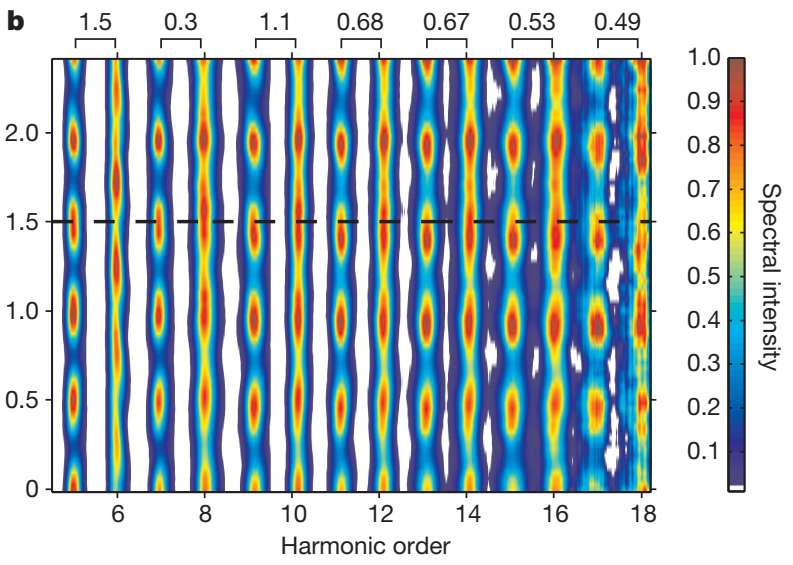

all harmonics modulate in phase. Each harmonic order is normalized separately. The relative intensity between even-order and adjacent odd-order harmonics is given at the top.

11. Vampa, G. et al. Theoretical analysis of high harmonic generation in solids. Phys. Rev. Lett. 113, 073901 (2014)

12. Hawkins, P. G., Ivanov, M. Y.\& Yakovlev, V. S. Effect of multiple conduction bands on high-harmonic emission from dielectrics. Phys. Rev. A 91, 013405 (2015).

13. Dudovich, N. et al. Measuring and controlling the birth of attosecond XUV pulses. Nature Phys. 2, 781-786 (2006)

14. He, X. et al. Interference effects in two-color high-order harmonic generation. Phys. Rev. A 82, 033410 (2010)

15. Ulstrup, S. et al. Ultrafast dynamics of massive Dirac fermions in bilayer graphene. Phys. Rev. Lett. 112, 257401 (2014)

16. Keldysh, L. Ionization in the field of a strong electromagnetic wave. Sov. Phys. JETP 20, 1307-1314 (1965)

17. Stuart, B. C. et al. Nanosecond-to-femtosecond laser-induced breakdown in dielectrics. Phys. Rev. B 53, 1749-1761 (1996).

18. Wörner, H. J., Bertrand, J. B., Kartashov, D. V., Corkum, P. B. \& Villeneuve, D. M. Following a chemical reaction using high-harmonic interferometry. Nature 466 , 604-607 (2010).

19. Smirnova, O. et al. High harmonics interferometry of multi-electron dynamics in molecules. Nature 460, 972-977 (2009).

20. Zaks, B., Liu, R. B. \& Sherwin, M. S. Experimental observation of electron-hole recollisions. Nature 483, 580-583 (2012)

21. Brunel, F. Harmonic generation due to plasma effects in a gas undergoing multiphoton ionization in the high-intensity limit. J. Opt. Soc. Am. B 7, 521-526 (1990).

22. Schultze, M. et al. Attosecond band-gap dynamics of silicon. Science $\mathbf{3 4 6}$ 1348-1352 (2014).

23. Schultze, M. et al. Controlling dielectric with the electric field of light. Nature $\mathbf{4 9 3}$, 75-78 (2012)

24. Vu, Q. T. et al. Light-induced gaps in semiconductor band-to-band transitions. Phys. Rev. Lett. 92, 217403 (2004).

25. Eremets, M. I. \& Troyan, I. A. Conductive dense hydrogen. Nature Mater. 10, 927-931 (2011)

26. Shafir, D. et al. Resolving the time when an electron exits a tunnelling barrier. Nature 485, 343-346 (2012)

27. Gertsvolf, M., Spanner, M., Rayner, D. M. \& Corkum, P. B. Demonstration of attosecond ionization dynamics inside transparent solids. J. Phys. At. Mol. Opt Phys. 43, 131002 (2010).

28. Schiffrin, A. et al. Optical-field induced currents in dielectrics. Nature 493, 70-74 (2013)

29. Schwierz, F. Graphene transistors. Nature Nanotechnol. 5, 487-496 (2010).

30. Krausz, F. \& Stockman, M. I. Attosecond metrology: from electron capture to future signal processing. Nature Photon. 8, 205-213 (2014).

Acknowledgements We thank A. Larameé from the Advanced Laser Light Source for technical support during the experiment and M. Clerici for lending some equipment We acknowledge financial support from the US AFOSR, NSERC, FRQNT, MDEIE and CFI.

Author Contributions G.V. and P.B.C. conceived the experiment; G.V., T.J.H. and N.T. performed the experiment; N.T. and B.E.S. developed the laser source; P.B.C. and F.L. supervised the experiment; C.R.M. and T.B. supervised the theoretical calculations; all authors contributed to the manuscript.

Author Information Reprints and permissions information is available at www.nature.com/reprints. The authors declare no competing financial interests. Readers are welcome to comment on the online version of the paper. Correspondence and requests for materials should be addressed to P.B.C. (paul.corkum@nrc.ca) or G.V. (gvamp015@uottawa.ca). 


\section{METHODS}

Experimental set-up. High harmonics are generated with a mid-infrared laser source centred around $3.76 \mu \mathrm{m}$ and with $19 \mu \mathrm{J}$ pulse energy. The pulse duration of $95 \mathrm{fs}$ is measured with a dispersion-free SHG FROG after the ZnO crystal. The mid-infrared wavelength is obtained by difference-frequency generation in a $400 \mu \mathrm{m}$ thick $\mathrm{AgGaS}_{2}$ (AGS) crystal between the signal and the idler of a commercial Optical Parametric Amplifier (Light Conversion Topas-HE) pumped by $4.5 \mathrm{~mJ}$ from a Ti-sapphire laser. The output of the OPA is further amplified in a beta barium borate (BBO) crystal pumped by $10 \mathrm{~mJ}$ of $800 \mathrm{~nm}$ pulses. The midinfrared beam is spatially filtered with a pin-hole and focused with an $f / 30$ spherical Ag mirror onto an epitaxially grown $500 \mathrm{~nm}$ thin film of a single crystal of wurtzite $\mathrm{ZnO}(0001)$ deposited on a $0.5 \mathrm{~mm}$ sapphire(0001) substrate. The optical axes of both crystals are aligned parallel to the laser $\boldsymbol{k}$-vector. We used the knifeedge technique to measure the size of the focal spot.

The second harmonic is generated in a $300 \mu \mathrm{m}$ AGS crystal optimized for type-I SHG right after the pin-hole to exploit the high intensity and high beam quality. The second-harmonic intensity is controlled by moving the AGS crystal closer to or farther from the pin-hole. The two beams are separated and then recombined before the focusing mirror with dichroic beam splitters (LaserOptick) that reflect the second harmonic. Its polarization is rotated with a broadband $\lambda / 2$ plate before recombination. The delay between the two colours is scanned with a PZT stage on the second-harmonic arm.

The high harmonics are refocused with an $\mathrm{Al}$ mirror into a visible-ultraviolet spectrometer from OceanOptics.

The theoretical model. To interpret the experiment, we use the theoretical framework developed in ref. 11. The nonlinear response of the solid is described by a two band model whose characteristics are chosen to be those of $\mathrm{ZnO}$. The model solves the semiconductor Bloch equations for the time-dependent band populations $n_{m}(\boldsymbol{k}, t)(m=v, c$ for the valence and conduction bands respectively) and for the interband polarization $p(\boldsymbol{k}, t)$, where $\boldsymbol{k}$ is the crystal momentum, for a single electron-hole pair. Coulomb interaction between different pairs, leading to coupling of different $k$-states ${ }^{31}$, is neglected. The band parameters and lattice constants are reported in ref. 32. The laser parameters are: frequency $\omega=0.0121$ a.u., peak field strength $F_{0}=0.0049$ a.u.

Extended Data Fig. 1 shows the population of the conduction band along the $\Gamma \mathrm{M}$ direction of the reciprocal space as a function of time. The laser field creates electron-hole pairs around each field extreme. They are then accelerated to high crystal momenta and back to the centre of the Brillouin zone in a process that repeats every half laser cycle.

The model naturally includes the sub-cycle exchange of the band populationthe analogue of the step-wise ionization in gases. However, in solids both the single-band (intraband) and the two-band (interband) mechanism bear the signature of the generalized electron-hole recollision.

Extended Data Fig. 2a, b shows the high-harmonic spectrum of the respective intraband and interband terms as a function of time. The spectra are obtained from a windowed Fourier transform of the intra- and interband currents, defined in ref. 11, which can be separately calculated by the model. A 0.35 cycles wide Blackman window is scanned across half a laser cycle. The resulting spectra are continuous because only one recollision event is allowed inside the temporal window. We apply a super-Gaussian spectral filter that does not affect above bandgap harmonics, but progressively filters lower harmonics. This is done because the temporal window is too narrow for the lower orders (and the fundamental frequency) and results in artificial broadening of these harmonics which ultimately masks the weak high-harmonic signal if the filter is not applied. Interband harmonics above the bandgap are approximately $10^{4}$ times stronger than intraband ones. For each harmonic photon energy, the maximum spectral intensity determines its time-of-emission (or spectral phase).

For comparison, the time of generalized recollision is traced in red in Extended Data Fig. 2a, b. The time of re-encounter of the electron with its associated hole is extracted from their classical motion in the conduction (for the electron) and valence (for the hole) bands relative to the time of the field crest in which they were created at zero crystal momentum by strong-field tunnelling. Their acceleration in reciprocal space is $\boldsymbol{k}(t)=\boldsymbol{A}(t)-\boldsymbol{A}\left(t^{\prime}\right)$, where $\boldsymbol{A}(t)$ is the laser vector potential, and $t^{\prime}$ is the time of creation of the electron-hole pair. The real space velocity is $\boldsymbol{v}_{m}[\boldsymbol{k}]=\nabla_{\boldsymbol{k}} E_{m}(\boldsymbol{k})$, with $E_{m}(\boldsymbol{k})$ the energy dispersion for band $m$, from which the instantaneous position is obtained by temporal integration. This semiclassical model, introduced in ref. 11, has been detailed in ref. 32 .

Extended Data Fig. 2 shows that both interband and intraband currents produce high harmonics with a spectral phase determined by the generalized recollision. This recollision contribution is contained in the structure of the intraband equation $^{11}$. Contrary to interband harmonics, intraband emission is dominated by nonlinear oscillations and by tunnelling below the 18th harmonic order. The transition is reflected in the in situ calculation (see Fig. 2), where $\Phi_{\text {osc }}$ becomes independent on the delay between fundamental and second harmonic below the 18th harmonic.

Many electron-hole pairs are created by the strong laser field. Owing to the interaction of one electron (hole) with the others, the electron (hole) can scatter to a different $k$-state than that of its correlated hole (electron), therefore preventing their recombination. However, in a many-body theory, each carrier can encounter a different oppositely charged partner. In atomic high harmonic generation it was argued that recombination of the electron with an uncorrelated hole is not allowed in principle ${ }^{33}$. This debate is yet to be settled in solids. Our experiment only detects recollision between correlated electron-hole pairs.

Modelling the experiment. A weak second-harmonic field is added to the fundamental one to simulate the in situ experiment. The high-harmonic spectrum is recorded for each phase between the two colours. The resulting spectrograms for the interband and intraband emission are reported in Extended Data Fig. 3a, b respectively. We require the second harmonic to be $6 \times 10^{-4}$ relative to the fundamental to produce even high harmonics that are $\sim 5 \%$ of the odd ones. The modulation depth is close to $100 \%$ for both intra- and interband generated above bandgap harmonics. It drops to $\sim 50 \%$ for below bandgap interband harmonics and for harmonics below the 18th for intraband emission. The spectrograms of the interband and intraband currents and of the sum of the two are analysed in the same way as the experimental data: (i) the intensity of each harmonic is spectrally integrated over its width; (ii) the resulting intensity modulation as a function of delay is offset to 0 and normalized to 1 and (iii) fitted to a cosine function of delay with form $\cos \left(\phi_{\text {delay }}+\Phi_{\text {osc }}\right)$. Finally, $\Phi_{\text {osc }}$ for both emission mechanisms and for the total emission (the sum of the two) is extracted and shown in Fig. 2 of the main text.

Comparison with atomic high-harmonic generation. We analyse the similarities between the high harmonics from atoms and solids based on calculation of classical trajectories. In Extended Data Fig. 4 we plot $\Phi_{\text {osc }}$ calculated for an isolated atom (green line). The field strength of the fundamental is increased to $0.44 \mathrm{~V}^{-1}$ to allow the same cut-off as in $\mathrm{ZnO}$. The phase for interband emission in $\mathrm{ZnO}$ and the experimental data are the blue line and the black circles, respectively. According to the analytical equivalence between atomic and solid emission, demonstrated in refs 11,32 , the intensity modulation of the even harmonics can be calculated starting from the additional dipole phase $^{13}$ :

$$
\sigma(t, \phi)=\int_{t^{\prime}(t)}^{t} v\left(\tau, t^{\prime}\right) \boldsymbol{A}_{2}(\tau, \phi) \mathrm{d} \tau
$$

Where $\boldsymbol{A}_{2}(\tau, \phi)=\boldsymbol{A}_{2} \cos (2 \omega \tau+\phi)$ is the vector potential of the second harmonic and $\boldsymbol{v}(\boldsymbol{k})=\nabla_{\boldsymbol{k}} \varepsilon_{g}(\boldsymbol{k})$, with $\varepsilon_{g}(\boldsymbol{k})$ the momentum dependent bandgap. Once the semi-classical trajectories are known (as discussed in the previous section), the integral can be computed and the phase of the modulation extracted from ${ }^{13}$ $\Phi_{\mathrm{osc}}=\operatorname{atan}\left(\sigma_{\mathrm{c}} / \sigma_{\mathrm{s}}\right)$ with $\sigma(t, \phi)=\sigma_{\mathrm{s}}(t) \cos (\phi)+\sigma_{\mathrm{c}}(t) \sin (\phi)$

In atomic high-harmonic generation there are two important contributions to a given high harmonic. One is from the so-called short trajectory electrons. They appear as the curve with negative slope at larger phases in Extended Data Fig. 4. Almost all research on gas-phase harmonics has concentrated on this contribution. There are also long trajectory electrons. They are responsible for the other part of the curve and are often ignored in gases since their divergence is larger and an experiment can be designed to minimize their impact.

In $\mathrm{ZnO}$, the long trajectory contribution is significantly different from the atomic case. This is a direct consequence of the non-parabolic band dispersion. The distortion of the trajectories is extensively analysed in ref. 32. Further, no prediction can be made for below-bandgap harmonics, since classically the electron cannot recollide with energy smaller than the minimum bandgap. A quantum mechanical calculation that includes the effect of the tunnelling step is required to extend the comparison to smaller photon energies.

Theoretical cut-off scaling of interband emission. The high-harmonic spectrum extends up to a defined maximum harmonic order, called the cut-off. In atoms, the cut-off is related to the maximum kinetic energy available to the electron when it recombines with the hole. The cut-off depends linearly on the ponderomotive energy $U_{\mathrm{p}}$, which, in turn, depends quadratically on the field strength.

In contrast with atomic harmonics, experiments in solids ${ }^{6}$ show a linear scaling of the cut-off with field strength. Theoretical investigation of the intraband current confirmed the linear scaling. Extended Data Fig. 5 shows that the simulated interband current also leads to a cut-off that scales linearly with the field strength. Exploiting the analytical solution for this current ${ }^{32}$, it is possible to derive a semiclassical approximate cut-off scaling law that fully agrees with the simulation. This analysis is reported in ref. 32 .

Role of interband emission for various field strengths. We theoretically investigate the relative importance of interband and intraband emission at different field strengths at the laser wavelength used in this experiment. Extended Data Fig. 6 reports the interband and intraband spectra for $F_{0}=0.003$ a.u., $F_{0}=0.0049$ a.u. 


\section{RESEARCH LETTER}

(corresponding to the field strength reached in our experiment) and $F_{0}=0.008$ a.u (corresponding to the field strength reached in ref. 6, including the loss from normal incidence reflection). In all cases, interband emission dominates by four orders of magnitude for harmonic orders above the minimum bandgap (marked by the vertical dashed black line). Below the minimum bandgap the difference is less. A less clear harmonic structure is observed at the highest field strength. The loss of contrast arises from the interference between two sets of recolliding trajectories: one of electrons born slightly after the peak of the field (the 'short' branch of the atomic case) with one of electrons born just before the peak of the field. In the atomic case, electrons born before the peak do not recollide. In a solid, the non-parabolic shape of the band structure allows these extra recombinations. The topic will be a matter of a future publication.

31. Golde, D., Kira, M., Meier, T. \& Koch, S. W. Microscopic theory of the extremely nonlinear terahertz response of semiconductors. Phys. Status Solidi B 248, 863-866 (2011).

32. Vampa, G., McDonald, C. R., Orlando, G., Corkum, P. B. \& Brabec, T. Semiclassica analysis of high harmonic generation in bulk crystals. Phys. Rev. B 91, 064302 (2015).

33. Niikura, $\mathrm{H}$. et al. Probing molecular dynamics with attosecond resolution using correlated wave packet pairs. Nature 421, 826-829 (2003). 


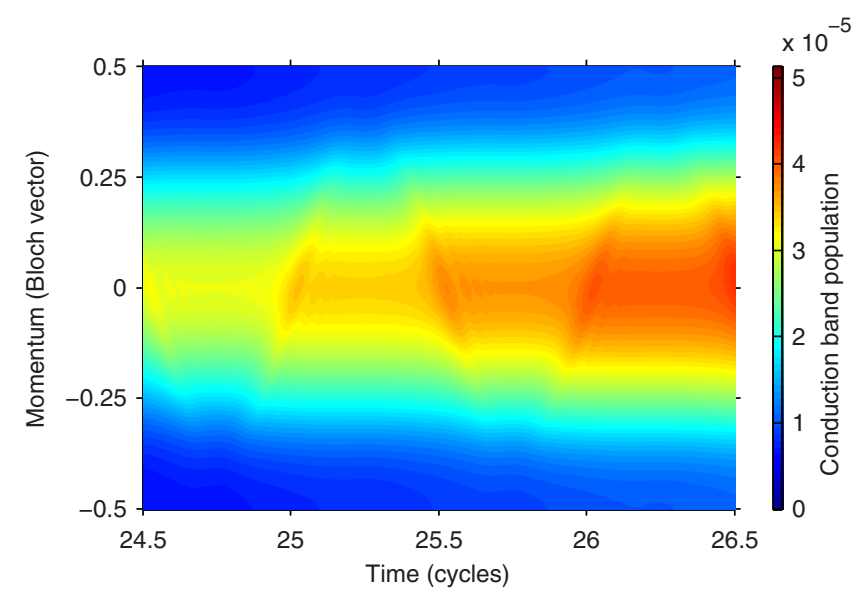

Extended Data Figure $1 \mid$ Evolution of conduction band population with time as a function of Bloch vector. Population in the conduction band (colour-coded, key at right) is created at each peak of the laser field and subsequently accelerated to large crystal momenta. The population is resolved along the $\Gamma \mathrm{M}$ direction of the Brillouin zone. 


\section{RESEARCH LETTER}

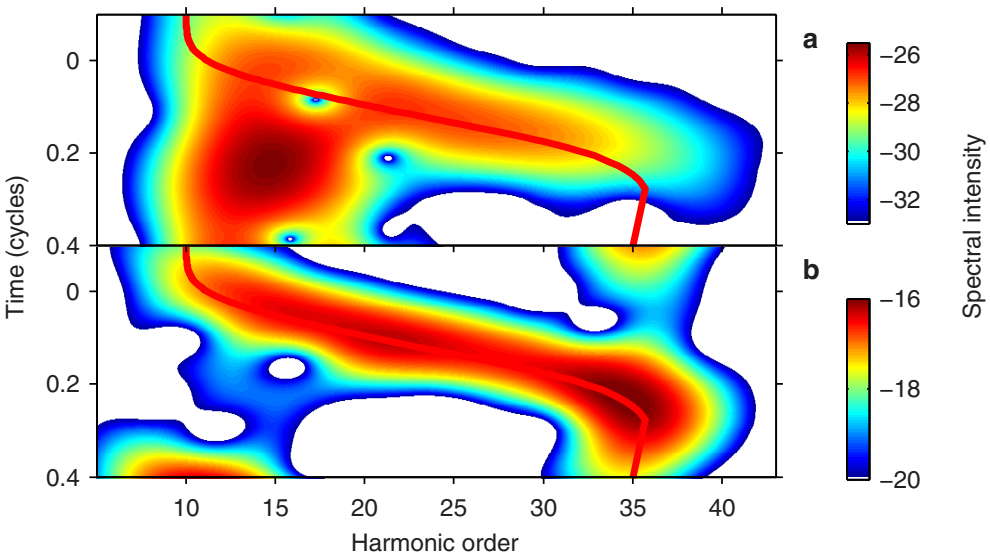

Extended Data Figure $2 \mid$ High-harmonic emission is timed to the electronhole recollision. The spectral content of the intraband (a) and interband

recollision between the electron and the hole (red line). The intensity (b) mechanisms as a function of time shows that each high harmonic is emitted at a specific moment of the laser cycle. This moment is the time of generalized (colour-coded) is on a logarithmic scale. The laser parameters are: $\omega=0.0121$ a.u., $F_{0}=0.0049$ a.u. 

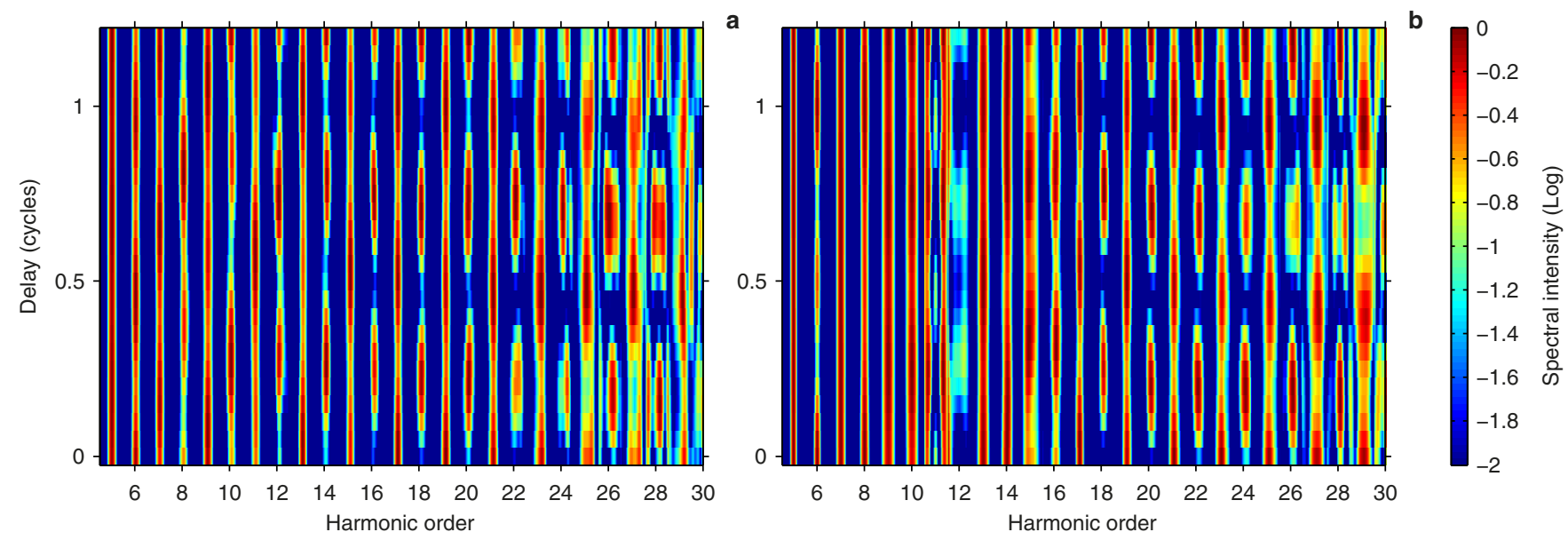

Extended Data Figure $3 \mid$ Predicted in situ spectrograms. Simulated spectrogram for interband (a) and intraband (b) emission. Intensity is colour-coded on a logarithmic scale. Each harmonic order has been normalized to simplify comparison. The laser parameters are: $\omega=0.0121$ a.u., $F_{0}=0.0049$ a.u., $F_{2 \omega}=10^{-2} F_{0}$. 


\section{RESEARCH LETTER}

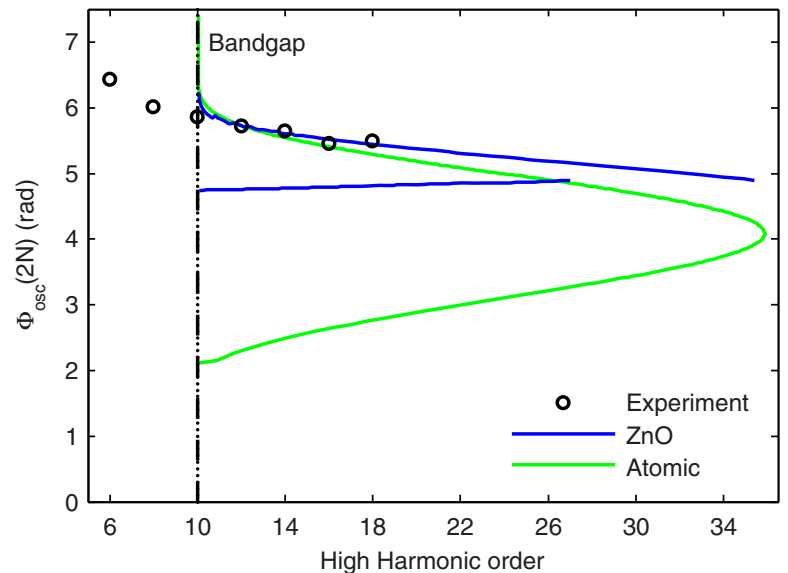

Extended Data Figure $4 \mid$ Comparison of atomic and solid high-harmonic generation. The phase of the modulation of the even harmonic intensity is plotted as a function of harmonic order. The green line is obtained for an atom with ionization potential equal to the bandgap of $\mathrm{ZnO}(3.3 \mathrm{eV}$, marked by the vertical black dashed line). The blue line and the black circles are the theoretical prediction for the interband source and the experimental data points, respectively. The theoretical prediction is based on classical trajectory calculation. 


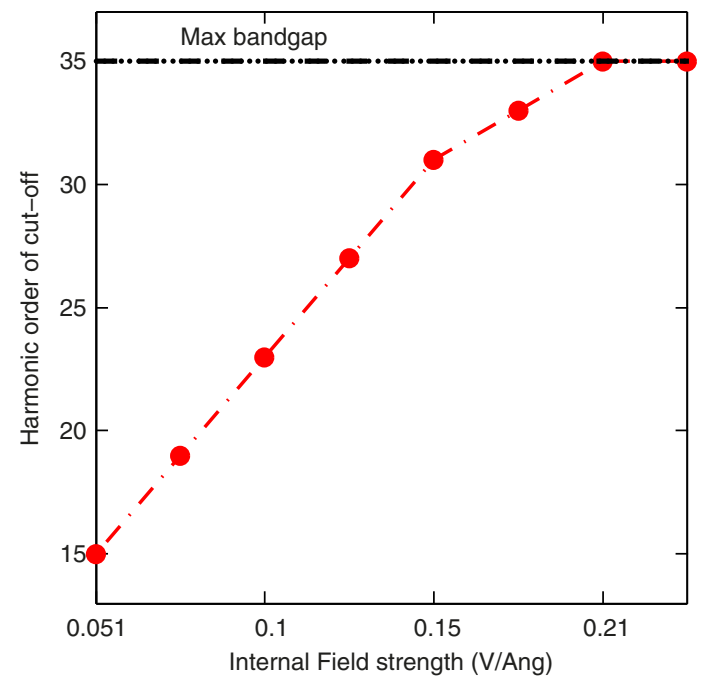

Extended Data Figure $5 \mid$ Cut-off scaling of interband emission. The highharmonic cut-off of interband emission predicted by the numerical solution of the two-band model is plotted as a function of field strength. The cut-off scales linearly with the field strength up to the maximum bandgap (reached at the edge of the Brillouin zone). The calculated values (red dots) are connected by the dashed dotted line to show the linear scaling. 


\section{RESEARCH LETTER}

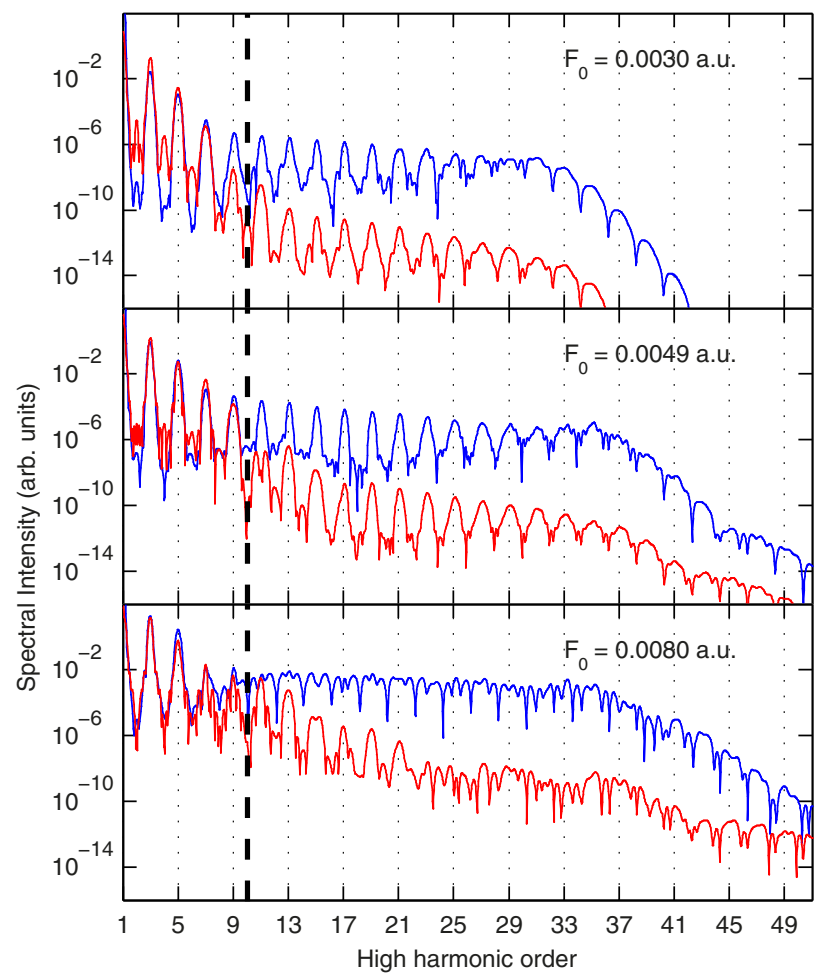

Extended Data Figure $6 \mid$ Scaling of high-harmonic emission with the field strength of the fundamental. The two-band model predicts that interband emission dominates over a wide range of field strengths. The field strength of our experiment is $F_{0}=0.0049$ a.u. (middle panel), while that of ref. 6 corrected for the reflection loss is $F_{0}=0.008$ a.u. (bottom panel). The vertical black dashed line marks the minimum bandgap (at $3.3 \mathrm{eV}$ ). 